\title{
Computer-Aided Contact-Less Localization of Latent Fingerprints in Low-Resolution CWL Scans
}

\author{
Andrey Makrushin ${ }^{1,2}$, Tobias Kiertscher ${ }^{2}$, Robert Fischer ${ }^{2}$, Stefan Gruhn², \\ Claus Vielhauer ${ }^{2}$, and Jana Dittmann ${ }^{1}$ \\ ${ }^{1}$ Otto-von-Guericke University of Magdeburg, Universitätsplatz 2, \\ 39106 Magdeburg, Germany \\ \{makrushin, jana.dittmann\}@iti.cs.uni-magdeburg.de \\ ${ }^{2}$ Brandenburg University of Applied Sciences, Magdeburger Str. 50, \\ 14770 Brandenburg an der Havel, Germany \\ \{kiertscher,fischerr, gruhns, vielhauer\} afh-brandenburg.de
}

\begin{abstract}
In forensic investigations, the recovering of latent fingerprints is one of the most essential issues. Driven by human experts, today this process is very time consuming. An automation of both examination of suspicious areas and acquisition of fingerprints lead on the one hand to the covering of larger surfaces and on the other hand to significant speed up of the evidence collection. This work presents an experimental study on capabilities of chromatic white-light sensor (CWL) regarding the contact-less localization of latent fingerprints on differently challenging substrates. The fully automatic CWL-based system is implemented from the acquisition through the feature extraction right up to the classification. The key objective of the work is to develop a methodological approach for the quantitative evaluation of the localization success. Based on the proposed performance measures, the optimal system parameters such as scan resolution, extracted features and classification scheme are specified dependent on the surface material. Our experiments from an actual project with the sensor industry partner show convincing localization performance on easy-to-localize and adequate performance on moderate-tolocalize substrates. The hard-to-localize substrates require further improvements of the localization system.
\end{abstract}

Keywords: Forensics, latent fingerprints, automated localization, contact-less acquisition, CWL sensor, quantitative evaluation.

\section{$1 \quad$ Introduction}

The standard way of recovering latent fingerprints from a crime scene is the superimposing of powder or chemical reagent to suspicious surfaces, which allows better visual distinguishing of ridge patterns from background, and the subsequent application of adhesive tape or conventional photo camera. The lifting of latent fingerprints is formerly considered a manual process for highly skilled forensic experts. The main disadvantage of the traditional procedure is its destructive nature. 
Chemical reagents destroy original traces, making further analysis (e.g. DNA extraction) impossible. Further, there are cases of special kinds of very fine or expensive objects (e.g. artworks) where any kind of intrusive contact is unacceptable. The manual fingerprint lifting is also time-consuming, so that experts normally investigate only selected areas of limited size. However, the potential fingerprint areas may be notably larger and a significant amount of finger traces may be lost.

A contact-less chromatic white-light (CWL) sensor has been originally developed for the surface quality assessment. The usage of a device for detection and acquisition of latent fingerprints is the novel idea and is worth careful research. This work presents an experimental study on capabilities of a CWL device to localize finger traces on differently challenging substrates. The work is done within a project with the sensor industry partner and includes the development of CWL-based imaging system (incl. data acquisition, feature extraction and classification) as well as the development of a methodological approach for the quantitative evaluation of the localization success. There are two concepts proposed: block-based and region-based evaluation. During the block-based evaluation, an acquired surface is divided into non-overlapping blocks and the algorithm decides for each block, whether it is a part of a fingerprint. The performance is given by the number of misclassified blocks. For the region-based evaluation, the positively classified blocks standing side by side are combined to potential fingerprint regions. These regions are compared to the original ones. As a measure of the correct localization the relative overlap between an original region and a discovered region is proposed. False positive responses are characterized by the total square of falsely found areas. The proposed performance measures allow the determination of optimal parameters such as scan resolution, block size, features to extract and classification scheme. The experiments carried out on the database of 560 fingerprints from 10 subjects. Due to complexity of the data gathering only three surface material are considered: white furniture, brushed metal and non-glossy car body finish. However, these materials are selected to represent groups of materials with similar physical properties.

Hereafter the paper is organized as follows. Section 2 gives an overview over related research. In Section 3 our localization system is presented in detail based on the work within a running project with the sensor industry partner. The experimental results are discussed in Section 4. Section 5 concludes the paper and gives an insight into further work.

\section{Related Works}

Traditional techniques for the detection of latent fingerprints are thoroughly described in the book of Champod et al. [1]. These techniques include the choice of chemical reagents and the corresponding treatments for porous, nonporous and semiporous surfaces to improve the visual perception of traces, or their visibility under alternative illuminations, e.g. in ultraviolet spectrum or in near-infrared spectrum. If a chemical treatment is not required, detection techniques are considered non-invasive. Nowadays several contact-less microscopic devices have been developed for noninvasive fingerprint acquisition. Leich et al. [2] gives an overview over these technologies with the focus on a CWL sensor. 
Since the outcome of a contact-less acquisition device is a kind of digital image, the localization of latent fingerprints can be interpreted as a texture analysis for the separation of objects from background. The localization task arises in various applications. The most known example is face localization, widely used in digital cameras. However, even for this well studied problem, the localization criterion is not uniformly defined [3]. Some authors consider the Euclidean distance between the centers of the detected and the true face and width proportions of both. Other authors consider the relation between found and original eyes positions. Therefore, the unambiguous measure of the localization success for fingerprints has to be defined. The traditional way to measure the detection success is a receiver operating characteristic (ROC) curve, which presents true detection rates in relation to false detection rates. Here, the location of the target in the image is assumed to be known or of no importance. For unknown-location tasks, where the precise localization of the targets is of crucial importance, the ROC is insufficient. Swensson [4] extends the ROC definition for the localization task. The localization response (LROC) curve, free-response (FROC) curve and alternative free-response (AFROC) curve have been introduced. The LROC measures not only the ability to detect, but also the ability to correctly localize targets in images with the assumption that each image contains only one target. The FROC and AFROC are designed for multiple-target images and give a fraction of all true positive reports against the mean number of false positive reports per image and against the abscissa of the ROC curve, correspondingly. Nevertheless, the direct usage of mentioned performance measures is hardly imaginable for the fingerprint localization, because the square of falsely detected area has more importance than the number of falsely recalled regions. Hence, the novel evaluation model including block-based and region-based evaluations is introduced in this paper.

\section{Our Fingerprint Localization System}

A CWL sensor provides point measurements of the proximity and luminance by means of a beam of white-light and the effect of chromatic aberration of lenses. The lenses focus wavelengths of the white-light at a different distance. The length of the wave reflected most strongly along with the focal length exactly matches the distance to the surface, so that the outcome represents the topography matrix. The amount of reflected light is recovered as well, resulting in the luminance matrix. Fries Research and Technology GmbH [5] has kindly provided a FRT MicroProf 200 device with a FRT CWL 600 sensor for our experiments. Regarding the manufacturer specification the lateral axis resolution can vary from 2 to $400 \mu \mathrm{m}$ and the vertical axis has a resolution of $20 \mathrm{~nm}$. The depth range amounts to $660 \mu \mathrm{m}$ for a single layer whereby multilayer scanning is also possible.

Following the concepts proposed by Hildebrandt et al. [6], Figure 1 introduces the fingerprint recovering workflow supported by CWL sensor. This paper addresses only the first CWL utilization scenario, namely contact-less non-intrusive localization of the areas with a structure similar to a fingerprint. Apart from the reliability, two requirements for the localization exist. It has to be done quickly and only lowresolution scans are permitted. The second demand is motivated by privacy 
preservation reasons. The FBI standard prescribes at least 500 dpi for reliable minutiae extraction. Jain et al. [7] specify resolutions from 250 to $300 \mathrm{dpi}$ as a minimum requirement allowing feature extraction algorithms to locate minutiae in a fingerprint image. Therefore, an image with the resolution lower than or equal to 250 dpi is determined here as coarse or low-resolution scan. Transferring this statement to properties of a CWL sensor, the lateral distance between two measurements has to be at least $100 \mu \mathrm{m}$. In experiments, three resolutions are considered: 100, 200 and 400 $\mu \mathrm{m}$. Following our concept, these low-resolution scans preserve privacy due to the impossibility to reliably extract minutiae.

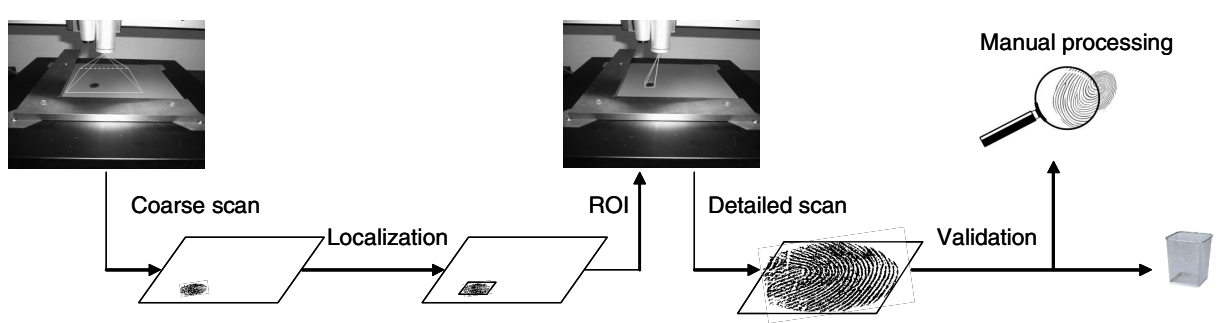

Fig. 1. Beginning with rapid acquisition and localization of suspicious regions using a coarse scan, the system provides the region of interest (ROI) for repeated scanning with a highresolution and successive validation of the fingerprint area. Hence, the forensic experts are expected to work only with a limited number of approved fingerprint regions.

The sliding window approach is the most common way of object localization. The window of particular size slides across an image, and resulted local blocks are processed independently. The processing implies extraction of features and subsequent classification of a block as the target or background. Considering this approach as a preliminary step, the localization of complex objects is done by connecting the positively recognized blocks to the regions and determining the joint boundary. In our system, blocks in the 8-connected neighborhood are merged to a single region. Resulted regions may be intersected, which is quite disadvantageous regarding the necessity for repeated acquisition of the same areas. Therefore, we propose two approaches for the processing of intersected regions shown in Figure 2.

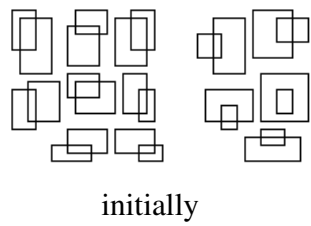

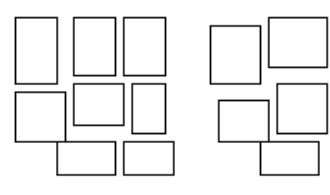

after "overcover"
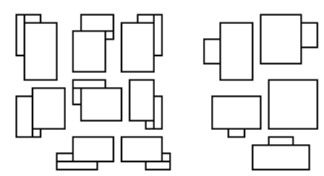

after "mosaic"

Fig. 2. Processing of intersected regions: The "overcover" approach merges all intersected regions to one minimal rectangular region enclosing any of those regions; The "mosaic" approach aims at minimization of the square of detected area through a tessellation of intersected regions. The larger region remains unchanged and the smaller region is split to two regions, so that one of two resulted regions has the maximal square. The outgoing regions with no positively classified blocks as well as insufficiently large regions are removed. 
Turning back to the separate block analysis in the framework of the sliding window approach, the processing begins with the feature extraction. Three feature groups are considered in this work: statistical, spectral and gradient-based features. The calculation of statistical features is motivated by the industrial standard [8]. The feature set consists of 9 measurements: maximum value, mean value, root mean square, variance, standard deviation, skewness, kurtosis, roughness and median value. The spectral features aim at calculation of direction and regularity of the ridge lines in the block. The approximate width of ridge lines fluctuates between $150 \mu \mathrm{m}$ and 800 $\mu \mathrm{m}$ depending on time and intensity of the finger contact with a surface. Therefore, three spectral bandwidths with the wave length intervals of 128-256, 256-512 and 512-1024 $\mu \mathrm{m}$ are taken into account. For each spectral bandwidth 9 statistical features in frequency domain are calculated (see [9] for details). The last feature group addresses the estimation of ridge properties in the block and contains three features: mean gradient, standard deviation of gradient and coherence [10]. The calculation of gradients is provided through the application of Sobel operators. After the feature extraction, each block is represented by a 39-dimensional feature vector.

According to Duda et al. [11], there is no universal classification technique, which is identically effective for all classification tasks. The decision to use one or another classifier can be done only for particular application. On this basis, several widely established machine learning concepts are examined in our study: decision trees (cart), support vector machine (svm-p1, svm-p2, svm-rbf), discriminant analysis (nblin, nb-quad, lda, qda) and $k$-nearest-neighbor rule (1nn, 5nn, 15nn, 31nn). Decision tree is the most intuitive way of the pattern classification. Here, the classification is done by answering successive questions, whereby each following question depends on the answer to the previous one. For the metric data this process can be interpreted as the consecutive creation of decision boundaries perpendicular to feature axes. SVM relies on the principle of structural risk minimization, meaning that the optimal separating hyper-plane is obtained as a trade-off between the empirical risk and the complexity of the classifier. This guarantees a high level of generalization ability. Therefore, SVM is effective for classification of high-dimensional data being in possession of small sets of examples. Furthermore, SVMs can solve linearly nonseparable problems using kernel functions. The discriminate analysis is a parametrical way of statistical model building and implicates several models for the fitting of the training data. Assuming that the data in different classes is generated by several Gaussian processes, the training is provided through estimating the parameters of a Gaussian distribution for each class. The class prediction for a test vector is done by the assignment of the class with the largest posterior probability. The $k-\mathrm{NN}$ is addressed as an example of a practically successful non-parametric classification technique, where the training degenerates to the collecting of training samples also called prototypes. The decision to place a test vector to one or another class is done through examining the labels of $k$ nearest prototypes and taking a vote. The detailed description of the classifiers can be found in [11]. All classifier implementations except SVM are taken from Matlab's Statistics Toolbox [12]. The SVM classification is based on the SVMLIB [13]. 


\section{$4 \quad$ Evaluation}

Since an empirical evaluation requires collecting a sufficiently large set of samples, only three substrates are examined: white furniture, brushed metal and non-glossy car body finish (see Table 1), aiming at determining overall sensor properties and a success tendency. In practice, most materials have similar properties regarding the traces appearance, so that the substrates are selected to represent material groups with the similar fingerprint detection complexity. The similar localization performance is expected for all members of a group. For each substrate, 560 fingerprints have been collected from 10 subjects. Finger traces have been deposited on the $15 \times 15 \mathrm{~cm}$ plate according to the predefined stencil mask. The stencil describes fingerprint regions by giving their coordinates and size. The scans have been done with three lateral measurement distances: 400, 200 and $100 \mu \mathrm{m}$. The 2-fold cross-validation with permutations is applied to obtain more statistically significant conclusions. The data from five persons is used for the training and from five remaining persons for the performance evaluation. Finally, each test includes 2800 fingerprints (56 fingerprints x 5 subjects $\mathrm{x} 10$ permutations).

Table 1. Representative materials

\begin{tabular}{|l|l|l|}
\hline Surface & Description & Luminance CWL-image \\
\hline furniture & $\begin{array}{l}\text { Smooth plastic material with clearly seen sweat } \\
\text { spots, due to low water absorbing properties and } \\
\text { uniform reflection. For this group sweat traces } \\
\text { appear clearly different from the background, so } \\
\text { that it is fairly easy to localize fingerprint regions. }\end{array}$ & $\begin{array}{l}\text { adsorbed, but residues left after a finger contact } \\
\text { partially sink in the surface concavities, so that } \\
\text { fingerprint patterns seem to be partially smeared } \\
\text { out. For this group it is moderately hard to localize } \\
\text { finger traces. }\end{array}$ \\
\hline $\begin{array}{l}\text { Brushed } \\
\text { metal }\end{array}$ & $\begin{array}{l}\text { Brushed metal surfaces have generally coarse } \\
\text { urainy structure. In our case the striated substrate is }\end{array}$ \\
\hline $\begin{array}{l}\text { Non- } \\
\text { glossy } \\
\text { car body } \\
\text { finish }\end{array}$ & $\begin{array}{l}\text { Finished car body does not absorb water, but has } \\
\text { irregular reflection properties, meaning that the } \\
\text { light sinks into the partially transparent material, } \\
\text { deflects and then reflects to unpredictable } \\
\text { directions. This leads to serious distortions of sweat } \\
\text { traces in acquired data. This group contains } \\
\text { materials, where the localization of fingerprints is } \\
\text { really hard. }\end{array}$
\end{tabular}


Table 2. Results of block-based evaluation (HTER in \%)

\begin{tabular}{|c|c|c|c|c|c|c|c|c|c|c|c|c|c|c|c|}
\hline mat. & block & res. & features & cart & $n b-\operatorname{lin}$ & nb-quad & Ida & qda & svm-p1 & svm-p2 & svm-rbf & $1 \mathrm{nn}$ & $5 \mathrm{nn}$ & $15 \mathrm{nn}$ & $31 \mathrm{nn}$ \\
\hline white & $2 \times 2$ & 400 & stat. & 18.63 & 7.62 & 5.95 & 5.05 & 6.32 & 7.30 & 7.37 & 6.82 & 13.27 & 9.81 & 8.65 & 8.26 \\
\hline \multirow[t]{23}{*}{ furniture } & \multirow[t]{11}{*}{$\mathrm{mm}$} & \multirow[t]{3}{*}{$\mu \mathrm{m}$} & spect. & 23.06 & 10.07 & 10.91 & 8.93 & 7.49 & 16.52 & 17.77 & 16.49 & 16.90 & 13.87 & 12.73 & 12.39 \\
\hline & & & grad. & 48.04 & 25.06 & 27.65 & 23.77 & 29.02 & 40.64 & 50.00 & 42.72 & 32.44 & 27.33 & 28.44 & 29.05 \\
\hline & & & st.+sp.+grad. & 17.87 & 7.75 & 5.85 & 4.99 & 6.76 & 7.41 & 9.19 & 7.50 & 12.95 & 9.50 & 8.63 & 8.21 \\
\hline & & 200 & stat. & 17.01 & 6.22 & 3.61 & 3.76 & 3.85 & 4.26 & 4.57 & 3.96 & 12.01 & 9.24 & 8.17 & 7.63 \\
\hline & & \multirow[t]{3}{*}{$\mu \mathrm{m}$} & spect. & 16.08 & 6.36 & 3.67 & 7.09 & 6.52 & 7.08 & 12.06 & 9.12 & 11.76 & 9.22 & 8.50 & 8.47 \\
\hline & & & grad. & 25.50 & 14.48 & 13.18 & 13.19 & 12.95 & 10.76 & 34.34 & 10.74 & 19.18 & 13.53 & 11.51 & 11.07 \\
\hline & & & st.+sp.+grad. & 16.19 & 6.07 & 3.59 & 3.50 & 3.03 & 4.66 & 7.24 & 4.93 & 12.05 & 9.36 & 8.54 & 8.17 \\
\hline & & 100 & stat. & 14.56 & 6.42 & 3.59 & 3.68 & 3.74 & 3.12 & 3.46 & 3.10 & 9.23 & 6.24 & 5.22 & 4.94 \\
\hline & & \multirow[t]{3}{*}{$\mu \mathrm{m}$} & spect. & 11.83 & 6.25 & 3.65 & 6.19 & 4.98 & 3.26 & 9.03 & 5.04 & 8.87 & 6.29 & 6.09 & 6.04 \\
\hline & & & grad. & 20.54 & 10.66 & 6.79 & 9.35 & 6.48 & 5.89 & 19.51 & 5.86 & 13.09 & 7.70 & 6.21 & 5.82 \\
\hline & & & st.+sp.+grad. & 13.84 & 6.09 & 3.52 & 3.35 & 2.66 & 3.14 & 4.70 & 3.26 & 9.20 & 6.36 & 5.67 & 5.45 \\
\hline & $4 \times 4$ & 400 & stat. & 6.32 & 5.07 & 2.07 & 2.31 & 2.00 & 1.63 & 1.91 & 1.71 & 3.23 & 2.17 & 1.84 & 1.81 \\
\hline & $\mathrm{mm}$ & $\mu \mathrm{m}$ & spect. & 11.38 & 14.12 & 6.61 & 13.46 & 7.75 & 5.09 & 50.00 & 5.70 & 9.24 & 5.84 & 5.16 & 4.98 \\
\hline & & & grad. & 15.20 & 11.08 & 8.60 & 9.71 & 8.13 & 6.85 & 10.79 & 6.90 & 11.55 & 8.45 & 7.56 & 7.17 \\
\hline & & & st.+sp.+grad. & 6.07 & 6.24 & 3.22 & 2.31 & 2.42 & 1.72 & 2.22 & 1.94 & 2.98 & 1.98 & 1.80 & 1.85 \\
\hline & & 200 & stat. & 6.16 & 4.81 & 1.12 & 2.33 & 1.17 & 0.93 & 1.38 & 1.11 & 2.03 & 1.20 & 1.12 & 1.05 \\
\hline & & $\mu \mathrm{m}$ & spect. & 4.77 & 12.87 & 3.42 & 12.20 & 3.91 & 3.11 & 50.00 & 4.27 & 3.71 & 2.96 & 2.66 & 2.69 \\
\hline & & & grad. & 10.17 & 9.90 & 5.02 & 8.52 & 4.89 & 4.51 & 10.59 & 4.49 & 7.39 & 5.20 & 4.81 & 4.48 \\
\hline & & & st.+sp.+grad. & 5.90 & 6.34 & 2.05 & 2.33 & 1.27 & 0.91 & 1.98 & 1.25 & 2.07 & 1.09 & 0.99 & 1.01 \\
\hline & & 100 & stat. & 7.12 & 5.06 & 1.39 & 1.85 & 1.27 & 1.15 & 1.70 & 1.35 & 2.18 & 1.40 & 1.21 & 1.26 \\
\hline & & $\mu \mathrm{m}$ & spect. & 3.59 & 12.08 & 2.63 & 11.56 & 2.29 & 2.61 & 50.00 & 4.44 & 2.34 & 1.66 & 1.70 & 1.78 \\
\hline & & & grad. & 6.09 & 8.01 & 2.58 & 6.55 & 2.24 & 2.42 & 8.69 & 2.48 & 4.33 & 2.11 & 1.76 & 1.83 \\
\hline & & & st.+sp.+grad. & 5.71 & 6.74 & 1.79 & 1.87 & 1.14 & 1.02 & 2.25 & 1.44 & 1.79 & 1.20 & 1.00 & 1.08 \\
\hline brushed & $2 \times 2$ & 400 & stat. & 36.82 & 22.28 & 22.87 & 21.59 & 25.40 & 32.97 & 36.20 & 34.31 & 32.87 & 29.14 & 27.78 & 27.45 \\
\hline metal & $\mathrm{mm}$ & $\mu \mathrm{m}$ & spect. & 38.84 & 22.41 & 22.65 & 21.70 & 21.58 & 35.60 & 36.70 & 36.13 & 34.17 & 30.91 & 29.09 & 28.28 \\
\hline & & & grad. & 49.63 & 45.99 & 46.73 & 47.05 & 46.53 & 50.00 & 50.00 & 50.00 & 49.30 & 49.27 & 49.24 & 49.52 \\
\hline & & & st.+sp.+grad. & 36.14 & 22.02 & 22.23 & 21.31 & 24.91 & 31.85 & 36.40 & 34.45 & 32.58 & 29.52 & 28.30 & 27.97 \\
\hline & & 200 & stat. & 26.99 & 15.46 & 15.14 & 13.75 & 13.64 & 11.27 & 12.17 & 11.43 & 22.34 & 15.93 & 13.22 & 12.23 \\
\hline & & $\mu \mathrm{m}$ & spect. & 25.54 & 13.82 & 13.48 & 12.67 & 19.02 & 11.09 & 11.56 & 11.56 & 21.30 & 15.42 & 12.87 & 11.80 \\
\hline & & & grad. & 47.98 & 39.52 & 40.69 & 40.83 & 41.08 & 50.00 & 50.00 & 50.00 & 47.10 & 45.52 & 45.77 & 46.20 \\
\hline & & & st.+sp.+grad. & 24.65 & 14.24 & 13.81 & 10.84 & 14.48 & 10.57 & 11.61 & 11.09 & 21.50 & 15.48 & 12.84 & 11.88 \\
\hline & & 100 & stat. & 30.92 & 16.77 & 15.69 & 15.98 & 15.77 & 14.16 & 16.98 & 14.52 & 25.12 & 20.38 & 17.83 & 16.46 \\
\hline & & $\mu \mathrm{m}$ & spect. & 28.17 & 15.22 & 14.16 & 14.52 & 20.33 & 13.58 & 15.50 & 14.51 & 24.16 & 19.48 & 16.84 & 15.89 \\
\hline & & & grad. & 42.55 & 36.52 & 38.81 & 32.28 & 32.33 & 45.48 & 49.03 & 45.59 & 39.08 & 36.50 & 35.31 & 34.68 \\
\hline & & & st.+sp.+grad. & 26.33 & 15.57 & 14.47 & 12.20 & 15.61 & 12.27 & 15.62 & 13.30 & 22.87 & 18.23 & 15.96 & 15.05 \\
\hline & $4 \times 4$ & 400 & stat. & 25.90 & 18.68 & 18.57 & 17.63 & 21.13 & 14.68 & 17.76 & 15.51 & 21.98 & 16.79 & 15.69 & 15.51 \\
\hline & $\mathrm{mm}$ & $\mu \mathrm{m}$ & spect. & 45.31 & 38.81 & 40.45 & 35.17 & 38.11 & 49.72 & 49.93 & 49.71 & 44.42 & 42.32 & 41.61 & 42.32 \\
\hline & & & grad. & 46.81 & 39.97 & 43.10 & 39.00 & 40.08 & 50.00 & 50.00 & 49.98 & 45.96 & 43.94 & 43.51 & 43.32 \\
\hline & & & st.+sp.+grad. & 24.78 & 18.04 & 16.33 & 15.78 & 19.28 & 12.89 & 26.35 & 14.49 & 20.81 & 16.16 & 15.39 & 15.08 \\
\hline & & 200 & stat. & 14.39 & 13.78 & 12.73 & 10.69 & 14.29 & 7.85 & 8.02 & 8.23 & 12.56 & 8.86 & 8.06 & 8.28 \\
\hline & & $\mu \mathrm{m}$ & spect. & 33.74 & 31.53 & 32.99 & 19.56 & 24.97 & 26.34 & 47.02 & 38.81 & 32.61 & 30.25 & 29.30 & 29.33 \\
\hline & & & grad. & 40.65 & 33.62 & 33.93 & 31.85 & 32.01 & 41.44 & 47.55 & 42.91 & 37.31 & 33.78 & 32.25 & 31.88 \\
\hline & & & st.+sp.+grad. & 13.75 & 15.40 & 15.10 & 7.86 & 12.39 & 6.19 & 9.86 & 7.47 & 11.67 & 8.09 & 7.95 & 7.96 \\
\hline & & 100 & stat. & 23.47 & 15.83 & 14.06 & 13.40 & 15.19 & 10.44 & 11.13 & 10.89 & 17.33 & 11.91 & 10.87 & 10.71 \\
\hline & & $\mu \mathrm{m}$ & spect. & 30.19 & 28.01 & 28.96 & 14.62 & 19.49 & 18.13 & 38.76 & 29.98 & 30.49 & 27.23 & 26.10 & 25.97 \\
\hline & & & grad. & 31.08 & 31.19 & 31.74 & 21.40 & 21.84 & 27.18 & 41.11 & 23.76 & 27.12 & 22.53 & 20.18 & 19.56 \\
\hline & & & st. + sp. & 18.26 & 18.78 & 20.58 & 9.85 & 10.81 & 7.96 & 16.38 & 9.65 & 14.51 & 11.07 & 10.66 & 10.65 \\
\hline & $2 \times 2$ & 400 & stat. & 47.22 & 46.54 & 45.09 & 43.29 & 42.30 & 0.12 & 50.00 & 50.04 & 46.31 & 46.68 & 47.91 & 8.68 \\
\hline & $\mathrm{mm}$ & $\mu \mathrm{m}$ & spect. & 49.24 & 45.94 & 45.55 & 46.39 & 45.87 & 50.00 & 50.00 & 50.04 & 47.94 & 48.48 & 49.12 & 49.75 \\
\hline & & & grad. & 49.92 & 87 & 49.19 & 48.99 & 48.67 & 50.00 & 50.00 & 50.00 & 49.91 & 50.09 & 49.96 & 50.03 \\
\hline & & & st. $+\mathrm{sp}$ & $\frac{9}{9}$ & 24 & 51 & 43.24 & 42.58 & 12 & 50.00 & 50.09 & 47.37 & 13 & 49.02 & 49.53 \\
\hline & & 200 & stat. & 50.93 & 52.38 & 51.92 & 51.96 & 48.12 & 52.98 & 52.87 & 53.06 & 51.47 & 52.49 & 52.79 & 52.85 \\
\hline & & & spect. & 51.70 & 53.22 & 53.21 & 53.11 & 51.97 & 53.11 & 53.06 & 53.16 & 51.47 & 52.55 & 52.85 & 53.01 \\
\hline & & & grad. & 49.89 & 47.51 & 47.27 & 47.07 & 47.48 & 50.00 & 50.00 & 50.00 & 50.04 & 49.73 & 49.71 & 50.01 \\
\hline & & & st. + sp. & & & 53.15 & 52.46 & 51.91 & 52.94 & 52.97 & 53.07 & 51.74 & 52.52 & 52.89 & 53.03 \\
\hline & & 100 & stat. & 42.33 & 34.46 & 34.80 & 34.03 & 33.58 & 41.22 & 43.14 & 41.16 & 39.75 & 39.44 & 39.02 & 38.79 \\
\hline & & $\mu \mathrm{m}$ & spect. & 41.91 & 34.62 & 34.48 & 34.33 & 34.33 & 41.83 & 43.33 & 41.15 & 39.23 & 38.97 & 38.66 & 38.48 \\
\hline & & & grad. & 49.29 & 48.25 & 49.15 & 44.52 & 45.26 & 50.00 & 50.00 & 50.00 & 48.63 & 48.83 & 49.18 & 49.64 \\
\hline & & & st.+sp.+grad. & 41.53 & 34.58 & 34.61 & 35.52 & 32.61 & 40.95 & 43.29 & 41.08 & 39.29 & 39.03 & 38.43 & 38.35 \\
\hline & $4 \times 4$ & 400 & stat. & 41.26 & 42.93 & 40.97 & 39.04 & 38.20 & 46.52 & 49.70 & 47.80 & 40.64 & 42.41 & 43.81 & 44.30 \\
\hline & $\mathrm{mm}$ & $\mu \mathrm{m}$ & spect. & 49.97 & 46.63 & 46.58 & 46.23 & 46.39 & 50.00 & 50.00 & 50.00 & 49.69 & 49.23 & 49.46 & 49.21 \\
\hline & & & grad. & 49.69 & 47.49 & 47.43 & 47.42 & 47.85 & 50.00 & 50.00 & 50.00 & 49.91 & 48.82 & 49.38 & 49.57 \\
\hline & & & st.+sp.+grad. & 41.22 & 42.86 & 42.79 & 39.42 & 38.89 & 46.29 & 50.14 & 48.79 & 45.21 & 44.89 & 45.94 & 46.33 \\
\hline & & 200 & stat. & 51.04 & 52.17 & 52.16 & 49.30 & 46.69 & 52.29 & 52.53 & 52.72 & 51.90 & 52.13 & 52.30 & 52.37 \\
\hline & & & spect. & 48.93 & 44.87 & 45.42 & 44.71 & 46.58 & 50.00 & 50.00 & 50.00 & 49.70 & 49.16 & 48.69 & 48.78 \\
\hline & & & grad. & 48.91 & 45.78 & 45.69 & 45.22 & 45.00 & 50.00 & 50.00 & 50.00 & 49.34 & 48.00 & 47.82 & 48.16 \\
\hline & & & st. + sp.t & 51.12 & 47.30 & 48.74 & 48.76 & 46.30 & 52.08 & 51.23 & 52.76 & 50.54 & 51.69 & 52.11 & 52.50 \\
\hline & & 100 & stat. & 40.70 & 31.76 & 32.52 & 29.78 & 30.57 & 35.32 & 38.80 & 35.41 & 36.66 & 35.39 & 34.81 & 34.13 \\
\hline & & & spect. & 47.99 & 46.11 & 46.18 & 42.89 & 43.61 & 49.88 & 50.00 & 50.00 & 47.01 & 47.15 & 47.63 & 47.85 \\
\hline & & & grad. & 46.53 & 45.61 & 45.42 & 38.53 & 38.63 & 49.86 & 50.00 & 49.93 & 44.92 & 43.27 & 42.48 & 43.29 \\
\hline & & & st.+sp.+grad. & 38.35 & 35.96 & 34.89 & 28.05 & 29.52 & 33.85 & 47.24 & 34.88 & 34.56 & 34.09 & 34.09 & 34.18 \\
\hline
\end{tabular}


For the block-based evaluation the standard I-type and II-type errors are employed, meaning that fingerprint blocks may be falsely recognized as empty blocks (false negative response) and vice versa (false positive response). The average value of these outcomes (half total error rate [HTER]) is used as the localization performance measure. The experimental results in Table 2 show that blocks of $4 \times 4 \mathrm{~mm}$ invariably lead to better performance compared to $2 \times 2 \mathrm{~mm}$ blocks. In fact, $2 \times 2 \mathrm{~mm}$ blocks are too small to reliably calculate statistical features. The higher the resolution is, the better recognition rates are, whereby the difference in performance between 200 and $100 \mu \mathrm{m}$ is negligible. Generally the best results are achieved with one of three classifiers: SVM with the linear kernel, LDA or QDA. Considering the white furniture and brushed metal, the SVM with the linear kernel is asserted to be the most appropriate classifier, because of the best HTER values for $4 \times 4 \mathrm{~mm}$ blocks. LDA and QDA are the best classifiers for non-glossy car body finish. However, the resulted error rates (HTER $>28 \%$ ) annihilate the superiority of one or another classifier or feature group. In case of white furniture, statistical features provide very low error rates, so that their fusion with other features only slightly improves or in some cases just impairs the performance. In case of brushed metal, the extraction of spectral features is paid off. They often have better discriminative power than statistical features. Consequently, feature fusion leads to superior results compared to the single feature groups. Gradient-based features are seldom as successful as other features and they generally fail in applications for brushed metal and non-glossy car body finish.

The results of block-based evaluation helps to identify the best parameters of the system, but do not give a plausible image of localization success. For this reason, the positively recognized blocks are connected to regions. The localization accuracy is given by relative intersection ratio between true and found fingerprint regions, and introduced as follows: $I=0.5 *\left(S_{12} / S_{1}+S_{12} / S_{2}\right)$. Here $S_{1}$ and $S_{2}$ denote the squares of the true and found regions, correspondingly. The intersection area of both regions is given by $S_{12}$. The intersection ratio is calculated for each true fingerprint region and takes on the values from the interval [0..1]. The value 1 implies the absolute match and the value 0 the non-overlapping. In order to avoid uncertainties during the calculation of $I$, the detected regions must not intersect, which is guaranteed by the region merging approaches from Section 3. In the experiment the "mosaic" algorithm is used. For correct region localization, $I$ is defined to be at least $60 \%$.

Table 3 shows the results of region-based evaluation. Only the best classifiers with the optimal combination of features are presented. The application of $2 \times 2 \mathrm{~mm}$ blocks leads to higher detection rates but larger falsely recalled area. For both white furniture and brushed metal the scan resolutions of $200 \mu \mathrm{m}$ leads to optimal results meaning the relationship between the number of missed fingerprints and the total square of falsely recalled areas. The application of $4 \times 4 \mathrm{~mm}$ blocks is preferable because the returned area does not exceed $1 \%$ of the overall investigated area. For non-glossy car body finish the feature set has to be drastically improved. The square of the currently recalled area makes the application of the automatic localization on this substrate useless. 
Table 3. Results of region-based evaluation

\begin{tabular}{|c|c|c|c|c|c|c|c|c|c|c|c|}
\hline subtratum & block & res. & fetures & HTER & classifier & fp_num & fp_det & fp_missed & missed \% & nonfp_det & nonfp_sq. \\
\hline white & \multirow[t]{3}{*}{$4 \times 4$} & 400 & stat. & $1.63 \%$ & svm_lin & 2800 & 2789 & 11 & $0.39 \%$ & 10 & $1.00 \%$ \\
\hline \multirow{5}{*}{ furniture } & & 200 & stat.+spect. & $0.90 \%$ & svm_lin & 2800 & 2788 & 12 & $0.43 \%$ & 5 & $0.17 \%$ \\
\hline & & 100 & stat.+spect.+grad. & $1.00 \%$ & $15 \mathrm{nn}$ & 2800 & 2768 & 32 & $1.14 \%$ & 0 & $0.00 \%$ \\
\hline & \multirow[t]{3}{*}{$2 \times 2$} & 400 & stat.+spect. & $4.99 \%$ & Ida & 2800 & 2788 & 12 & $0.43 \%$ & 9 & $0.55 \%$ \\
\hline & & 200 & stat.+spect. & $2.98 \%$ & qda & 2800 & 2794 & 6 & $0.21 \%$ & 9 & $0.26 \%$ \\
\hline & & 100 & stat.+spect. & $2.65 \%$ & qda & 2800 & 2790 & 10 & $0.36 \%$ & 9 & $0.32 \%$ \\
\hline \multirow{6}{*}{$\begin{array}{l}\text { brushed } \\
\text { metal }\end{array}$} & \multirow[t]{3}{*}{$4 \times 4$} & 400 & stat.+spect.+grad. & $12.89 \%$ & svm_lin & 2800 & 2658 & 142 & $5.07 \%$ & 138 & $15.00 \%$ \\
\hline & & 200 & stat.+spect.+grad. & $6.19 \%$ & svm_lin & 2800 & 2653 & 147 & $5.25 \%$ & 22 & $0.97 \%$ \\
\hline & & 100 & stat.+spect.+grad. & $7.96 \%$ & svm_lin & 2800 & 2566 & 234 & $8.36 \%$ & 39 & $3.51 \%$ \\
\hline & \multirow[t]{3}{*}{$2 \times 2$} & 400 & stat.+spect. & $21.25 \%$ & Ida & 2800 & 2624 & 176 & $6.29 \%$ & 142 & $11.92 \%$ \\
\hline & & 200 & stat.+spect.+grad. & $10.57 \%$ & svm_lin & 2800 & 2753 & 47 & $1.68 \%$ & 55 & $6.84 \%$ \\
\hline & & 100 & stat.+spect.+grad. & $12.20 \%$ & Ida & 2800 & 2607 & 193 & $6.89 \%$ & 33 & $5.40 \%$ \\
\hline \multirow{6}{*}{$\begin{array}{l}\text { non-glossy } \\
\text { car body } \\
\text { finish }\end{array}$} & \multirow[t]{3}{*}{$4 \times 4$} & 400 & stat.+spect.+grad. & $38.89 \%$ & qda & 2800 & 2030 & 770 & $27.50 \%$ & 54 & $73.61 \%$ \\
\hline & & 200 & spect. & $44.71 \%$ & Ida & 2800 & 2239 & 561 & $20.04 \%$ & 32 & $93.71 \%$ \\
\hline & & 100 & stat.+spect.+grad. & $28.05 \%$ & Ida & 2800 & 2507 & 293 & $10.46 \%$ & 32 & $83.29 \%$ \\
\hline & \multirow[t]{3}{*}{$2 \times 2$} & 400 & stat. & $42.30 \%$ & qda & 2800 & 2076 & 724 & $25.86 \%$ & 104 & $62.19 \%$ \\
\hline & & 200 & grad. & $47.07 \%$ & Ida & 2800 & 2701 & 99 & $3.54 \%$ & 10 & $100.00 \%$ \\
\hline & & 100 & stat.+spect.+grad. & $32.61 \%$ & qda & 2800 & 2463 & 337 & $12.04 \%$ & 41 & $76.19 \%$ \\
\hline
\end{tabular}

\section{Conclusion}

Within a research project with the sensor industry partner, the development of the automatic contact-less CWL-based localization of latent fingerprints is introduced along with experimental evaluation on three differently challenging substrates. The novel evaluation approach is presented including block-based evaluation for the determination of optimal system parameters (scan resolution, block size, features to extract and classifier) and region-based evaluation to give a plausible impression of the localization success. In the best cases, the localization performance on easy-tolocalize substrate is $0.90 \%$ HTER, on moderate-to-localize substrate $6.19 \%$ and on hard-to-localize $-28.05 \%$. These results allow considering the proposed system to be very promising for forensic applications. Future work will be devoted to the improvement of features, the integration of the introduced system into the forensic framework and the development of fingerprint validation techniques in detailed CWL scans.

Acknowledgments. The work has been funded by the German Federal Ministry of Education and Science (BMBF) through the research program under the contract no. FKZ: 13N10818 and FKZ: 13N10816.

\section{References}

1. Champod, C., Lennard, C.J., Margot, P., Stoilovic, M.: Fingerprints and Other Ridge Skin Impressions. CRC Press, Boca Raton (2004)

2. Leich, M., Kiltz, S., Dittmann, J., Vielhauer, C.: Non-destructive forensic latent fingerprint acquisition with chromatic white light sensors. In: Proc. SPIE, vol. 7880, p. 78800S (2011)

3. Rodriguez, Y., Cardinaux, F., Bengio, S., Mariéthoz, J.: Measuring the performance of face localization systems. Image and Vision Computing Journal 24(8), 882-893 (2006) 
4. Swensson, R.G.: Measuring Detection and Localization Performance. In: Barrett, H.H., Gmitro, A.F. (eds.) IPMI 1993. LNCS, vol. 687, pp. 525-541. Springer, Heidelberg (1993)

5. Fries Research \& Technology GmbH, http: / /www. frt-gmbh. com/en

6. Hildebrandt, M., Dittmann, J., Pocs, M., Ulrich, M., Merkel, R., Fries, T.: Privacy Preserving Challenges: New Design Aspects for Latent Fingerprint Detection Systems with Contact-Less Sensors for Future Preventive Applications in Airport Luggage Handling. In: Vielhauer, C., Dittmann, J., Drygajlo, A., Juul, N.C., Fairhurst, M.C. (eds.) BioID 2011. LNCS, vol. 6583, pp. 286-298. Springer, Heidelberg (2011)

7. Jain, A., Chen, Y., Demirkus, M.: Pores and Ridges: Fingerprint Matching Using Level 3 Features. In: Proc. 18th Int. Conf. on Pattern Recognition, vol. 4, pp. 477-480 (2006)

8. ANSI/ASME B46.1-2009: Surface Texture, Surface Roughness, Waviness and Lay (2010)

9. Kiertscher, T., Fischer, R., Vielhauer, C.: Latent fingerprint detection using a spectral texture feature. In: Proc. 13th ACM Multimedia Workshop on Multimedia and Security (2011)

10. Fischer, R., Vielhauer, C.: Ausgewählte Biometrieverfahren zur Fingerspurdetektion. In: Horster, P. (ed.) Proc. D-A-CH Security, pp. 374-385 (2011)

11. Duda, R.O., Hart, P.E., Stork, D.G.: Pattern Classification, 2nd edn. Wiley Interscience (2000)

12. Matlab: Statistics Toolbox, http://www. mathworks.com/products/statistics

13. LIBSVM: A Library for Support Vector Machines, http://www.csie.ntu.edu.tw/ cjlin/libsvm 